\title{
A VERSION OF RUNGE'S THEOREM FOR THE HELMHOLTZ EQUATION WITH APPLICATIONS TO SCATTERING THEORY*
}

\author{
by R. L. OCHS, JR
}

(Received 20th July 1987)

\section{Introduction}

Let $D$ be a bounded, simply connected domain in the plane $R^{2}$ that is starlike with respect to the origin and has $C^{2, \alpha}$ boundary, $\partial D$, described by the equation in polar coordinates

$$
\rho=\rho(\phi), \quad 0 \leqq \phi \leqq 2 \pi,
$$

where $C^{2, \alpha}$ denotes the space of twice Hölder continuously differentiable functions of index $\alpha$. In this paper, it is shown that any solution $u \in C^{2, \alpha}(\bar{D})$ of the Helmholtz equation

$$
\Delta u+k^{2} u=0
$$

in $D$ can be approximated in the space $C^{1}(\bar{D})$ by an entire Herglotz wave function

$$
v(\rho, \phi)=\int_{0}^{\eta} g(\theta) \exp [i k \rho \cos (\theta-\phi)] d \theta, \quad \mathbf{x}=\rho e^{i \phi} \in R^{2}
$$

with kernel $g \in L^{2}[0,2 \pi]$ having support in an interval $[0, \eta]$ with $\eta$ chosen arbitrarily in $0<\eta<2 \pi$.

The reader will recall that an entire Herglotz wave function $v$ is a solution of the Helmholtz equation (1.1) in $R^{2}$ satisfying the boundedness condition

$$
\varlimsup_{r \rightarrow \infty} \frac{1}{r} \int_{|\mathbf{x}|<r}|v(\mathbf{x})|^{2} d \mathbf{x}<\infty .
$$

Wave functions satisfying (1.2) were first discussed by Herglotz in a lecture in 1945 [12], and the results of this lecture were published for the first time in 1952 by Müller [11] with considerable amplification. Hartman and Wilcox [10] proved that $v$ is an entire

*This work was supported in part by the Air Force Office of Scientific Research grant 860087. 
Herglotz wave function if and only if $v$ has the integral representation in polar coordinates $(\rho, \phi)$

$$
v(\rho, \phi)=\int_{0}^{2 \pi} g(\theta) \exp [i k \rho \cos (\theta-\phi)] d \theta
$$

where $g \in L^{2}[0,2 \pi]$ is uniquely determined and is called the Herglotz kernel for $v$.

In the final section of this paper, the approximation result mentioned above is used to present a method for solving the limited aperture problem of inverse acoustic scattering for scattering by a sound-hard obstacle. The solution scheme to be presented is based on the orthogonal projection approach of Colton and Monk $([4,5,6,7,8])$. This technique was used by Ochs [14] to solve, with numerical examples, the limited aperture problem for scattering by a sound-soft obstacle.

\section{The approximation theorem}

Before proving that we can approximate in $C^{1}(\bar{D})$ any solution $u \in C^{2, \alpha}(\vec{D})$ of the Helmholtz equation by an entire Herglotz wave function $v$, where the kernel of $v$ has support in $[0, \eta], 0<\eta \leqq 2 \pi$, we first prove the following theorem on the continuous dependence of $\nabla u$ on $u$. Since a theorem is available [14] stating that every solution $u \in C^{2 . \alpha}(\bar{D})$ can be uniformly approximated in $C(\bar{D})$ by a Herglotz wave function with kernel having support in $[0, \eta]$, then we will be done.

Theorem 2.1. Let $D$ be a bounded, simply connected, starlike domain where $\partial D$ is described by $\rho=\rho(\phi), 0 \leqq \phi \leqq 2 \pi$. Assume that $\rho$ is periodic with period $2 \pi$ and $\rho \in C^{2, \alpha}(-\infty, \infty)$. Let $\varepsilon>0$ be given. Then for all $u_{1}$ and $u_{2}$ solutions of the Helmholtz equation in $D$ such that $u_{1}, u_{2} \in C^{2, \alpha}(\bar{D})$, there exists $\delta>0$ such that if

$$
\max _{\mathbf{x} \in \tilde{D}}\left|u_{1}(\mathbf{x})-u_{2}(\mathbf{x})\right| \leqq \delta
$$

then

$$
\max _{\mathbf{x} \in \bar{D}}\left\|\nabla u_{1}(\mathbf{x})-\nabla u_{2}(\mathbf{x})\right\| \leqq \varepsilon
$$

where $\|\cdot\|$ denotes the ordinary Euclidean norm on vectors in $R^{2}$.

Proof. According to [16, p. 59], we may define in $\bar{D}$ harmonic functions $\mathrm{T} u_{i}, i=1,2$, given by

$$
\left(\mathbf{T} u_{i}\right)(r, \theta)=u_{i}(r, \theta)+\int_{0}^{r} u_{i}(p, \theta) \frac{r}{\rho} \frac{\partial}{\partial r} J_{0}(k \sqrt{\rho(\rho-r)}) d \rho .
$$

We note that if $w_{i}:=\mathbf{T} u_{i}$, then 


$$
u_{i}(r, \theta)=\left(\mathbf{T} w_{i}\right)(r, \theta)=w_{i}(r, \theta)-\int_{0}^{r} w_{i}(\rho, \theta)(\partial / \partial \rho) J_{0}(k \sqrt{r(r-\rho)}) d \rho .
$$

By $\left[15\right.$, p. 15 , Theorem 6], for $\delta>0$ there exists a starlike domain $D_{0} \supset \supset \bar{D}$ and harmonic functions $h_{i} \in C^{2, \alpha}\left(\bar{D}_{0}\right)$ such that

$$
\max _{\mathbf{x} \in \bar{D}}\left|h_{i}(\mathbf{x})-\left(\mathbf{T} u_{i}\right)(\mathbf{x})\right| \leqq \delta
$$

and

$$
\max _{\mathbf{x} \in \bar{D}}\left\|\nabla h_{i}(\mathbf{x})-\nabla\left(\mathbf{T} u_{i}\right)(\mathbf{x})\right\| \leqq \delta, \quad i=1,2
$$

Clearly, if

$$
\max _{\mathbf{x} \in \bar{D}}\left|u_{1}(\mathbf{x})-u_{2}(\mathbf{x})\right| \leqq \delta
$$

then there exists a $C>0$ such that

$$
\max _{\mathbf{x} \in \bar{D}}\left|\left(\mathbf{T} u_{1}\right)(\mathbf{x})-\left(\mathbf{T} u_{2}\right)(\mathbf{x})\right| \leqq C \delta,
$$

since $T$ is a bounded operator. Combining inequalities (2.1) and (2.4) we can conclude via the triangle inequality that

$$
\max _{\mathbf{x} \in \bar{D}}\left|h_{1}(\mathbf{x})-h_{2}(\mathbf{x})\right| \leqq(C+2) \delta .
$$

Using the Heine-Borel Theorem and the continuity of $h_{1}$ and $h_{2}$ in $\bar{D}_{0}$, we can conclude that there exists a simply connected domain $D_{*}$ with smooth boundary such that $D \subset \subset D_{*} \subset \subset D_{0}$ and

$$
\max _{\mathbf{x} \in \bar{D}_{*}}\left|h_{1}(\mathbf{x})-h_{2}(\mathbf{x})\right| \leqq 2(C+2) \delta
$$

Let $G_{D}(\mathbf{x} ; \xi)$ be the Green function for the Dirichlet problem for Laplace's equation in $D_{*}$. Then

$$
h_{i}(\mathbf{x})=\int_{\partial D_{*}} \frac{\partial G_{D}}{\partial n_{\xi}}(\mathbf{x} ; \xi) h_{i}(\xi) d s(\xi), \quad i=1,2, \quad \mathbf{x} \in D_{*}
$$

So

$$
\nabla h_{1}(\mathbf{x})-\nabla h_{2}(\mathbf{x})=\int_{\partial D_{*}} \nabla_{\mathbf{x}} \frac{\partial G_{D}}{\partial n_{\xi}}(\mathbf{x} ; \xi)\left[h_{1}(\xi)-h_{2}(\xi)\right] d s(\xi)
$$


Thus from (2.5) and (2.6), we can conclude that there exists a $C^{\prime}>0$ such that

$$
\max _{\mathbf{x} \in \overline{\mathbf{D}}}\left\|\nabla h_{1}(\mathbf{x})-\nabla h_{2}(\mathbf{x})\right\| \leqq 2 C^{\prime}(C+2) \delta
$$

Using the triangle inequality on (2.2) and (2.7) we conclude that

$$
\max _{\mathbf{x} \in \bar{D}}\left\|\nabla\left(\mathbf{T} u_{1}\right)(\mathbf{x})-\nabla\left(\mathbf{T} u_{2}\right)(\mathbf{x})\right\| \leqq\left[2 C^{\prime}(C+2) \delta+2\right] \delta
$$

So, denoting partial differentiation with respect to $r$ and $\theta$ (which are the polar coordinates of $\mathbf{x}$ ) by subscripts, there exists $K>0$ such that

$$
\max _{\mathrm{x}=(r, \theta) \in \bar{D}}\left|\left(\mathrm{~T} u_{1}\right)_{r}(r, \theta)-\left(\mathrm{T} u_{2}\right)_{r}(r, \theta)\right| \leqq K \delta
$$

and

$$
\max _{\mathbf{x}=(r, \theta) \in \bar{D}}\left|\left(\mathbf{T} u_{1}\right)_{\theta}(r, \theta)-\left(\mathbf{T} u_{2}\right)_{\theta}(r, \theta)\right| \leqq K \delta
$$

Let $\mathbf{T} u_{i}=w_{i}, i=1,2$. Then

$$
u_{i}(r, \theta)=w_{i}(r, \theta)-\int_{0}^{r} w_{i}(\rho, \theta) \frac{\partial}{\partial \rho} J_{0}(k \sqrt{r(r-\rho)}) d \rho
$$

and

$$
u_{1}(r, \theta)-u_{2}(r, \theta)=w_{1}(r, \theta)-w_{2}(r, \theta)-\int_{0}^{r}\left[w_{1}(\rho, \theta)-w_{2}(\rho, \theta)\right] \frac{\partial}{\partial \rho} J_{0}(k \sqrt{r(r-\rho)}) d \rho .
$$

So,

$$
\left|u_{1_{\theta}}(r, \theta)-u_{2_{\theta}}(r, \theta)\right| \leqq\left|w_{1_{\theta}}(r, \theta)-w_{2_{\theta}}(r, \theta)\right|+\int_{0}^{r}\left|w_{1_{\theta}}(\rho, \theta)-w_{2_{\theta}}(\rho, \theta)\right|\left|\frac{\partial}{\partial \rho} J_{0}(k \sqrt{r(r-\rho)})\right| d \rho
$$

and

$$
\begin{aligned}
\left|u_{1_{r}}(r, \theta)-u_{2_{r}}(r, \theta)\right| \leqq & \left|w_{1_{r}}(r, \theta)-w_{2_{r}}(r, \theta)\right|+\left|w_{1}(r, \theta)-w_{2}(r, \theta)\right|\left[\frac{\partial}{\partial \rho} J_{0}(k \sqrt{r(r-\rho)})\right]_{\rho=r} \mid \\
& +\int_{0}^{r}\left|w_{1}(\rho, \theta)-w_{2}(\rho, \theta)\right|\left|\frac{\partial^{2}}{\partial r \partial \rho} J_{0}(k \sqrt{r(r-\rho)})\right| d \rho .
\end{aligned}
$$

We must now prove that 


$$
\frac{\partial}{\partial \rho} J_{0}(k \sqrt{r(r-\rho)}) \text { and } \frac{\partial^{2}}{\partial r \partial \rho} J_{0}(k \sqrt{r(r-\rho)})
$$

remain bounded as $\rho \rightarrow 0^{+}$and $\rho \rightarrow r^{-}$.

First note that $([2$, p. 1])

$$
J_{1}(z)=\frac{z}{2}+0\left(z^{3}\right)
$$

and

$$
J_{1}^{\prime}(z)=\frac{1}{2}-\frac{3}{16} z^{2}+0\left(z^{4}\right)
$$

for all $|z|$ small. Now,

$$
\begin{aligned}
\frac{\partial}{\partial \rho} J_{0}(k \sqrt{r(r-\rho)}) & =J_{0}^{\prime}(k \sqrt{r(r-\rho)}) \cdot \frac{-k r}{2 \sqrt{r(r-\rho)}} \\
& =J_{1}(k \sqrt{r(r-\rho)}) \cdot \frac{k r}{2 \sqrt{r(r-\rho)}}
\end{aligned}
$$

([1, p. 361]). Thus, by $(2.8),(\partial / \partial \rho) J_{0}(k \sqrt{r(r-\rho)})$ is uniformly bounded for all $\rho \in(0, r)$. Also,

$$
\frac{\partial^{2}}{\partial r \partial \rho} J_{0}(k \sqrt{r(r-\rho)})=J_{1}^{\prime}(k \sqrt{r(r-\rho)}) \cdot \frac{k^{2} r(2 r-\rho)}{4 r(r-\rho)}+J_{1}\left(k \sqrt{r(r-\rho))}\left(\frac{-k r \rho}{4 r(r-\rho) \sqrt{r(r-\rho)}}\right) .\right.
$$

By equation (2.9)

$$
J_{1}^{\prime}(k \sqrt{r(r-\rho)})=\frac{1}{2}-\frac{3 k^{2} r(r-\rho)}{16}+0\left(k^{4} r^{2}(r-\rho)^{2}\right)
$$

So,

$$
J_{1}^{\prime}\left(k \sqrt{r(r-\rho))} \frac{k^{2} r(2 r-\rho)}{4 r(r-\rho)}=\frac{k^{2} r(2 r-\rho)}{8 r(r-\rho)}-\frac{3 k^{4} r^{2}(r-\rho)(2 r-\rho)}{64 r(r-\rho)}+0\left(r^{2}(r-\rho)\right)\right.
$$

and

$$
J_{1}(k \sqrt{r(r-\rho)})\left(\frac{-k r \rho}{4 r(r-\rho) \sqrt{r(r-\rho)}}\right)=\frac{-k^{2} r \rho}{8 r(r-\rho)}+0(\rho)
$$

which imply that 


$$
\begin{aligned}
\frac{\partial^{2}}{\partial r \partial \rho} J_{0}(k \sqrt{r(r-\rho)}) & =\frac{k^{2} r(2 r-\rho)-k^{2} r \rho}{8 r(r-\rho)}+0(1) \\
& =\frac{2 k^{2} r(r-\rho)}{8 r(r-\rho)}+0(1)=0(1) .
\end{aligned}
$$

Therefore, there exists $C^{\prime \prime}>0$ such that

$$
\max _{\mathbf{x} \in \bar{D}}\left\|\nabla u_{1}(\mathbf{x})-\nabla u_{2}(\mathbf{x})\right\| \leqq C^{\prime \prime} \delta .
$$

So, given $\varepsilon>0$, chosen $\delta=\varepsilon / C^{\prime \prime}$, which completes the proof of the theorem.

Now we combine this with

Theorem 2.2. [14]. Let $\eta \in(0,2 \pi]$ be given, and let $u$ be a solution of the Helmholtz equation in a bounded, simply connected, starlike domain $D$ where $\partial D$ is described by $\rho=\rho(\phi), 0 \leqq \phi \leqq 2 \pi$. Assume that $\rho$ is periodic with period $2 \pi, \rho \in C^{2, \alpha}(-\infty, \infty)$, and $u \in C^{2, \alpha}(\bar{D})$. Then for every $\varepsilon>0$ there exists an entire Herglotz wave function $v$ with kernel having support in $[0, \eta]$ such that

$$
\max _{\mathbf{x} \in \bar{D}}|u(\mathbf{x})-v(\mathbf{x})| \leqq \varepsilon
$$

Hence the main result of this section.

Theorem 2.3. Let $\eta \in(0,2 \pi]$ be given and let $D$ and $u$ be as above. Then for every $\varepsilon>0$ there exists an entire Herglotz wave function $v$ with kernel having support in $[0, \eta]$ such that

$$
\max _{\mathbf{x} \in \bar{D}}|u(\mathbf{x})-v(\mathbf{x})| \leqq \varepsilon
$$

and

$$
\max _{\mathbf{x} \in \bar{D}}\|\nabla u(\mathbf{x})-\nabla v(\mathbf{x})\| \leqq \varepsilon
$$

\section{Applications to scattering theory}

In this section, the utility of Theorem 2.3 is demonstrated by using it is construct a scheme for solving the inverse scattering problem for a sound-hard obstacle. Let us state the problem precisely.

Let $D$ be a bounded, simply connected domain in the plane with Hölder continuously 
differentiable boundary $\partial D$ and let the field incident on $D, u^{i}$, be given by $u^{i}(\mathbf{x})=$ $\exp [i k \mathbf{x} \cdot \boldsymbol{\alpha}]$, where $\mathbf{x} \in R^{2}, k>0$ is the wave number, and $\boldsymbol{\alpha}$ is a fixed unit vector giving the direction of propagation of $u^{i}$. Denote the field scattered by the obstacle $D$ by $u^{s}$, and define the total field $u$ by $u=u^{i}+u^{s}$. Then the direct scattering problem for an acoustically hard obstacle $D$ is to find a solution $u \in C^{2}\left(R^{2} \backslash \bar{D}\right) \cap C^{1}\left(R^{2} \backslash D\right)$ of the Helmholtz equation

$$
\Delta_{2} u+k^{2} u=0
$$

in $R^{2} \backslash \bar{D}$ such that

$$
\frac{\partial u}{\partial v}=0 \quad \text { on } \quad \partial D
$$

and $u^{s}$ satisfies the Sommerfeld radiation condition

$$
\lim _{r \rightarrow \infty} r^{1 / 2}\left(\frac{\partial u^{s}}{\partial r}-i k u^{s}\right)=0
$$

uniformly in all directions. Here $\Delta_{2}$ is the two-dimensional Laplacian operator, $r=|\mathbf{x}|$, and $\partial / \partial v$ denotes differentiation in the direction of the outward normal $v$ to $\partial D$. (The direct scattering problem for a sound-soft obstacle results if condition (3.2) is replaced by

$$
u=0 \quad \text { on } \quad \partial D \text {. }
$$

This problem is dealt with in [14].) It is easily shown [3] that $u^{s}=u-u^{i}$ has the asymptotic bhavior

$$
u^{s}(r, \theta)=\frac{e^{i k r}}{\sqrt{r}} F(\theta ; k, \alpha)+0\left(r^{-3 / 2}\right)
$$

where $(r, \theta)$ is the polar representation of a point $\mathbf{x}$ in the plane and $F$ is known as the far field pattern for the scattered wave $u^{s}$. The inverse problem we consider here is to determine $\partial D$ given $F\left(\theta ; k, \alpha_{n}\right)$ for all $\theta$ such that $0 \leqq \theta \leqq \eta$, where $\eta$ is any positive number less than or equal to $2 \pi, k$ takes on all values in an interval of the positive real axis to be prescribed later and $\alpha_{n}, n=1, \ldots, N$, are $N$ distinct unit vectors. We make the assumption without loss of generality that the far field patterns $F\left(\theta ; k, \alpha_{n}\right), n=1, \ldots, N$, are given for all $\theta$ in the interval $[0, \eta]$ as opposed to a more general interval $[a, a+\eta] \subset[0,2 \pi]$, since a rotation can always be performed.

From Green's formula and the asymptotic behavior of the Hankel function $H_{0}^{(1)}$ of the first kind of order zero, one easily shows that [3] 


$$
F(\theta ; k, \alpha)=\frac{e^{i \pi / 4}}{\sqrt{8 \pi k}} \int_{\partial D} u(\mathbf{y}) \frac{\partial}{\partial v(y)} \exp [-i k \rho \cos (\theta-\phi)] d s(\mathbf{y})
$$

where $\mathbf{x}=r e^{i \theta}, \mathbf{y}=\rho e^{i \phi}, v$ is the unit outward normal to $\partial D$, and $u^{s}=u-u^{i}$ is the unique solution of a Fredholm integral equation of the second kind [3]. Clearly, the relationship between $\partial D$ and the far field pattern $F$ is nonlinear, and $F(\theta ; k, \alpha)$ is an analytic function of the argument $\theta$. So the inverse scattering problem for a sound-hard obstacle is ill-posed, and we reformulate the problem as a nonlinear optimization problem such that a priori information about the boundary of the obstacle can be used to stabilize the problem.

So, let $g \in L^{2}[0, \eta]$ be arbitrary and return to the representation (3.5) of the far field pattern. Then we have

$$
\int_{0}^{\eta} F(\theta ; k, \boldsymbol{x}) \overline{g(\theta)} d \theta=\frac{e^{i \pi / 4}}{\sqrt{8 \pi k}} \int_{\partial D} u(\mathbf{y}) \frac{\partial}{\partial v(y)} \overline{v(\mathbf{y} ; k)} d s(\mathbf{y})
$$

where

$$
v(\mathbf{y} ; k)=\int_{0}^{\eta} g(\theta) \exp [i k \rho \cos (\theta-\phi)] \mathrm{d} \theta
$$

is an entire Herglotz wave function with Herglotz kernel having support in $[0, \eta]$. Note that if $v$ is an interior Neumann eigenfunction of $D$, then the right hand side of (3.6) vanishes showing that the Herglotz kernel $g$ is orthogonal to $F$ in $L^{2}[0, \eta]$. This observation, together with Theorem 2.3, suggests a way of formulating the optimization problem. Define the minimization problem

$$
\mu(F)=\min _{\left(k^{2}, \boldsymbol{g}, \rho\right)} \varepsilon U\left\{\sum_{n=1}^{N}\left|\int_{0}^{n} F\left(\theta ; k, \boldsymbol{\alpha}_{n}\right) \overline{g(\theta)} d \theta\right|^{2}+\int_{0}^{2 \pi}\left|\frac{\partial}{\partial v_{\rho}} v(\rho(\phi), \phi ; k)\right|^{2} d \phi\right\}
$$

where

$$
v(\rho, \phi, k):=\int_{0}^{\eta} g(\theta) \exp [i k \rho \cos (\theta-\phi)] \mathrm{d} \theta
$$

$\left(\partial / \partial v_{\rho}\right)$ denotes differentiation along the outward normal to the closed curve $\rho=\rho(\phi)$, and $U$ is a compact set of ordered triples $\left(k^{2}, g, \rho\right)$ over which the minimization is performed. For example, $U=I^{C} \times U_{1}^{C} \times U_{2}^{c}$. Here $I^{C}$ is a compact set of positive real numbers containing the (unknown!) first nonzero eigenvalue of the Laplacian for Neumann boundary conditions in $\partial D: \rho=\rho(\phi) . U_{1}^{c}$ is a compact set of functions $g \in L^{2}[0, \eta]$ satisfying $\int_{0}^{\eta}|g(\theta)|^{2} d \theta \leqq M^{2}$ and suitably normalized to prevent $v(\rho, \phi ; k)$ from being trivial (see the appendix), and $U_{2}^{C}$ is a compact set of functions $\rho \in C^{1}[0,2 \pi]$ which are periodic with period $2 \pi$ and nonnegative. The latter set incorporates a priori constraints on trial boundaries, such as 


$$
0<a \leqq \rho(\phi) \leqq b \text { for all } \phi \in[0,2 \pi]
$$

and

$$
\max _{0 \leqq \phi \leqq 2 \pi}\left|\rho^{\prime}(\phi)\right| \leqq C,
$$

where $a, b$ and $C$ are known positive real numbers. We expect the expression in brackets in equation (3.7) to attain a minimum when $k^{2}$ equals the first nonzero Neumann eigenvalue of $D, g$ is the Herglotz kernel of the Herglotz wave function $v$ which is an approximation to the first interior Neumann eigenfunction itself, and $\rho=\rho(\phi)$ is a parametric representation of $\partial D$. The reason that the first nonzero eigenvalue is considered is that the eigenfunction corresponding to the zero eigenvalue is a constant and contains no information about $\partial D$. That this eigenfunction is unsuitable is clear from equation (3.7).

Now, it is clear that a solution exists to problem (3.7) since a continuous functional is being minimized over a compact set. A boundary parameterization $\rho \in C^{1}[0,2 \pi]$ corresponding to the third element of such a minimizing ordered triplet will be called an admissible solution of the inverse scattering problem. Theorems are now presented showing the continuous dependence of the admissible solutions on the far field data and that if the boundary parameterization of the actual solution of the inverse scattering problem is within the set of trial boundaries, then $\mu(F, M, N, \tau) \rightarrow 0$ as $M \rightarrow \infty$. Recall that $N$ is the number of plane waves incident on the obstacle and $M$ is the upper bound on the $L^{2}$-norm of all functions $g \in U_{1}^{c} . \tau$ is defined in the appendix.

Theorem 3.1. Let $\Phi(F)$ be the set of admissible solutions corresponding to the far field pattern $F$. Then if $F_{j} \rightarrow F$ in $L^{2}[0, \eta], \rho_{j} \in \Phi\left(F_{j}\right)$, then there exists a convergent subsequence of $\left\{\rho_{j}\right\}$, and every limit point lies in $\Phi(F)$. That is, $\Phi(F)$ is graph compact.

Theorem 3.2. Suppose $\partial D$, represented by $\rho=\rho(\phi)$, is a solution of the inverse scattering problem with the far field pattern $F$ such that $\rho \in C^{2, \alpha}[0,2 \pi] \cap U_{2}^{C}$. Assume that for $g$ a polynomial in $e^{i \theta}$ in $[0, \eta]$ and $\sum_{j=-\tau}^{\tau}\left|g_{j}\right|^{2}=1, \tau \geqq 0$ (see the appendix), we have that $g \in U_{1}^{C}$ for $M$ sufficiently large. Then for every $N$

$$
\lim _{M \rightarrow \infty} \mu(F, M, N, \tau)=0,
$$

for $\tau$ sufficiently large.

The proofs of these theorems are entirely analogous to those for the Dirichlet case [14] and will not be presented here.

Of course, it would be desirable to have a converse to Theorem 3.2, i.e., if $F$ is a far field pattern for a bounded, starlike domain $D$ and $\lim _{M \rightarrow \infty} \mu(F, M, N, \tau)=0$ for all $\tau$ sufficiently large, then the admissible functions $\rho_{M}$ tend to a limiting function $\rho$ and $\partial D: \rho=\rho(\phi)$ is a solution of the inverse scattering problem. But such a result is not true. 
Indeed, we cannot even obtain a partial converse, as we could in the Dirichlet case, asserting that all admissible solutions $\rho^{*}$ of problem (3.7) lie "outside" the actual solution $\partial D: \rho=\rho(\phi)$ of the inverse scattering problem in the sense that $\rho^{*}(\phi) \geqq \rho(\phi)$ for all $\phi \in[0,2 \pi]$. This is clear from the following example for the full aperture problem $(\eta=2 \pi)$.

Example. Let $F$ be the far field pattern corresponding to the incoming plane wave $u^{i}(\mathbf{x})=\exp \left[i k \mathbf{x} \cdot \alpha_{n}\right]$, where $\alpha_{n}=\left(\cos \phi_{n}, \sin \phi_{n}\right)$ is an arbitrary unit vector and the scattering obstacle is the disc $|\mathbf{x}|<a$. Let $g_{1}(\theta)=\sqrt{2} \sin \theta$ and $g_{2}(\theta)=\sqrt{2} \cos \theta$. Then by separation of variables, the asymptotic behavior of the Hankel functions $H_{n}^{(1)}$, and the Jacobi-Anger expansion

$$
e^{i r \cos \theta}=\sum_{\eta=-\infty}^{\infty} i^{n \eta} J_{n}(r) e^{i n \theta}
$$

we have that

$$
\begin{gathered}
\int_{0}^{2 \pi} F\left(\theta ; k, \alpha_{n}\right) \overline{g_{1}(\theta)} d \theta=-2 \sqrt{2} \pi e^{-i \pi / 4} \sqrt{\frac{2}{\pi k}} \frac{J_{1}^{\prime}(k a)}{H_{1}^{(1)}(k a)} \sin \phi_{n}, \\
\int_{0}^{2 \pi} F\left(\theta ; k, \alpha_{n}\right) \overline{g_{2}(\theta)} d \theta=-2 \sqrt{2} \pi e^{-i \pi / 4} \sqrt{\frac{2}{\pi k}} \frac{J_{1}^{\prime}(k a)}{H_{1}^{(1)}(k a)} \cos \phi_{n}, \\
v_{1}(\rho, \phi ; k):=\int_{0}^{2 \pi} g_{1}(\theta) \exp [i k \rho \cos (\theta-\phi)] d \theta=2 \sqrt{2} \pi i J_{1}(k \rho) \sin \phi, \\
\frac{\partial v_{1}}{\partial \rho}(\rho, \phi ; k)=2 \sqrt{2} \pi k i J_{1}^{\prime}(k \rho) \sin \phi, \\
\frac{\partial v_{1}}{\partial \phi}(\rho, \phi ; k)=2 \sqrt{2} \pi i J_{1}(k \rho) \cos \phi, \\
v_{2}(\rho, \phi ; k):=\int_{0}^{2 \pi} g_{2}(\theta) \exp [i k \rho \cos (\theta-\phi)] d \theta=2 \sqrt{2} \pi i J_{1}(k \rho) \cos \phi, \\
\frac{\partial v_{2}}{\partial \rho}(\rho, \phi ; k)=2 \sqrt{2} \pi k i J_{1}^{\prime}(k \rho) \cos \phi,
\end{gathered}
$$

and

$$
\frac{\partial v_{2}}{\partial \phi}(\rho, \phi ; k)=-2 \sqrt{2} \pi i J_{1}(k \rho) \sin \phi
$$


Let $k_{1 j}, j=1,2, \ldots$ be all the positive zeros of the Bessel function $J_{1}^{\prime}$ ordered so that $0<k_{11}<k_{12}<\ldots$. If $M \geqq \sqrt{2 \pi}$ and $\tau \geqq 1$, then $g_{i} \in U_{1}^{c}, i=1,2$, and for every integer $N$ we have that for $U$ appropriately chosen (in particular, $k^{2}=\left(k_{11} / a\right)^{2}=[$ first positive eigenvalue for the Neumann problem in the disc of radius $\left.a] \in I^{T}\right), \mu(F, M, N, \tau)=0$ for $\rho=\left(k_{1 m} / k_{11}\right) a$ for all $m=1,2, \ldots$ But the situation is more complicated than this. If one considers the collection of equations (3.10), (3.12), (3.13), and (3.14), one sees readily that a semicircle of radius $a$ is an admissible solution. The same conclusion can be drawn from equations (3.11), (3.15), (3.16), and (3.17), except that now the semicircles are rotated $90^{\circ}$ with respect to those in the previous sentence.

Note that the example indicates that semicircles are Herglotz domains, that is, domains having an interior eigenfunction (Neumann or Dirichlet boundary conditions) which is a Herglotz wave function [9]. In fact, by looking at the nodal curves and curves along which the normal derivative vanishes of the functions $u_{0}=J_{0}(k r), u_{m}=$ $J_{m}(k r) \sin m \theta, \tilde{u}_{m}=J_{m}(k r) \cos m \theta$ for all $m=1,2, \ldots$, one can find a generous supply of Herglotz domains.

Acknowledgement. This work appeared as part of a doctoral dissertation [13] written at the University of Delaware under the direction of Professor David L. Colton, to whom the author is greatly indebted.

\section{Appendix. Normalization of Neumann eigenfunctions}

Let $g \in L^{2}[0, \eta]$ be arbitrary and extend its domain of definition to $[0,2 \pi]$ by setting $g(\theta)=0$ for all $\theta$ in $(\eta, 2 \pi]$. Suppose that the obstacle $D$ contains the disc $\{\mathbf{x}:|\mathbf{x}|<a\}$ of positive radius $a$, and that

$$
v(\rho, \phi ; k)=\int_{0}^{2 \pi} g(\theta) \exp [i k \rho \cos (\theta-\phi)] d \theta
$$

is in interior Neumann eigenfunction corresponding to the first positive eigenvalue of the Laplacian in $D$. Then letting $\sum_{j=-\infty}^{\infty} g_{j} e^{i j \theta}$ be the Fourier series representation of $g(\theta)$, we get for $\rho<a$,

$$
\begin{aligned}
v(\rho, \phi ; k) & =\int_{0}^{2 \pi} g(\theta) \exp [i k \rho \cos (\theta-\phi)] d \theta \\
& =\sum_{j=-\infty}^{\infty} g_{j} \int_{0}^{2 \pi} e^{i j \theta} \exp [i k \rho \cos (\theta-\phi)] d \theta .
\end{aligned}
$$

Using the Jacobi-Anger expansion

$$
e^{i r \cos \theta}=\sum_{n=-\infty}^{\infty} i^{n} J_{n}(r) e^{i n \theta}
$$


we see that

$$
\begin{aligned}
\int_{0}^{2 \pi} e^{i j \theta} \exp [i k \rho \cos (\theta-\phi)] d \theta & =\int_{0}^{2 \pi} e^{i j \theta} \sum_{n=-\infty}^{\infty}(i)^{n} J_{n}(k \rho) e^{i n(\theta-\phi)} d \theta \\
& =\sum_{n=-\infty}^{\infty}(i)^{n} J_{n}(k \rho) e^{-i n \phi} \int_{0}^{2 \pi} e^{i j \theta} e^{i n \theta} d \theta \\
& =2 \pi(i)^{j} J_{j}(k \rho) e^{i j \phi}, \quad j=0, \pm 1, \pm 2, \ldots
\end{aligned}
$$

Thus

$$
v(\rho, \phi ; k)=\sum_{j=-\infty}^{\infty} 2 \pi(i)^{j} g_{j} J_{j}(k \rho) e^{i j \phi}=\sum_{j=-\infty}^{\infty} a_{j} J_{j}(k \rho) e^{i j \phi}, \quad \rho<a
$$

where $a_{j}:=2 \pi i g_{j}$. Clearly, if $v$ is to be nontrivial, there exists $n_{0} \geqq 0$ such that $a_{0}, a_{1}, a_{-1}, \ldots, a_{n_{0}}, a_{-n_{0}}$ are not all zero. So, for an integer $\tau \geqq n_{0}$, we normalize $g$ so that

$$
\sum_{j=-\tau}^{\tau}\left|g_{j}\right|^{2}=1
$$

\section{REFERENCES}

1. M. Aвramowitz and I. A. Stegun, Handbook of Mathematical Functions with Formulas, Graphs, and Mathematical Tables (Dover, New York, 1972).

2. F. Bowman, Introduction to Bessel Functions (Dover, New York, 1958).

3. D. Colton and R. Kress, Integral Equation Methods in Scattering Theory (John Wiley, New York, 1983).

4. D. Colton and P. Monk, A novel method for solving the inverse scattering problem for time harmonic acoustic waves in the resonance region, SIAM J. Appl. Math. 45 (1985), 1039-1053.

5. D. Colton and P. Monk, A novel method for solving the inverse scattering problem for time harmonic acoustic waves in the resonance region II, SIAM J. Appl. Math. 46 (1986), 506-523.

6. D. Colton and P. Monk, The numerical solution of the three dimensional inverse scattering problem for time harmonic acoustic waves, SIAM J. Sci. Statist. Comput. 8 (1987), 278-291.

7. D. Colton and P. Monk, The inverse scattering problem for time harmonic acoustic waves in a penetrable medium, Quart. J. Mech. Appl. Math. 40 (1987), 189-212.

8. D. Colton and P. Monk, The inverse scattering problem for time harmonic acoustic waves in an inhomogeneous medium, Quart. J. Appl. Math., 41 (1988), 97-125.

9. D. Colton and J. Wimp, Generalized Herglotz domains, Math. Meth. Appl. Sci. 8 (1986), 451-457. 
10. P. Hartman and C. Wilcox, On solutions of the Helmholtz equation in exterior domains, Math. Z. 75 (1961), 228-255.

11. C. Müller, Über die ganzen Lösungen der Wellengleichung, Math. Ann. 124 (1952), 235-264.

12. C. Müller, Angewandte Mathematik, Gustav Herglotz: Gesammelte Schriften (Hans Schwerdtfeger, ed., Vandenhoeck and Ruprecht, Göttingen, xxix-xxxi, 1979).

13. R. L. Ochs, J $\mathrm{J}_{\mathrm{R}}$, The limited aperture problem of inverse acoustic scattering (Ph.D. dissertation, University of Delaware, Newark, Delaware, 1986).

14. R. L. OchS, JR., The limited aperture problem of inverse acoustic scattering: Dirichlet boundary conditions, SIAM J. Appl. Math. 47 (1987), 1320-1341.

15. O. Pironneau, Optimal Shape Design for Elliptic Systems (Springer-Verlag, New York, 1984).

16. I. N. Vekua, New Methods for Solving Elliptic Equations (North-Holland, Amsterdam 1967).

Department of Mathematics

The UnIVERSITy OF Toledo

TOLEDO, Оhоо 43606

USA 